DIVISION OF THE HUMANITIES AND SOCIAL SCIENCES

CALIFORNIA INSTITUTE OF TECHNOLOGY

PASADENA, CALIFORNIA 91125

CORE MANY-TO-ONE MATCHINGS BY FIXED-POINT METHODS

Federico Echenique

California Institute of Technology

Jorge Oviedo

Universidad Nacional de San Luís

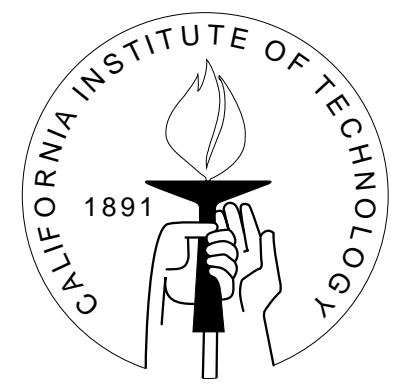

SOCIAL SCIENCE WORKING PAPER 1140 


\title{
Core Many-to-one Matchings by Fixed-Point Methods
}

\author{
Federico Echenique Jorge Oviedo
}

\begin{abstract}
We characterize the core many-to-one matchings as fixed points of a map. Our characterization gives an algorithm for finding core allocations; the algorithm is efficient and simple to implement. Our characterization does not require substitutable preferences, so it is separate from the structure needed for the non-emptiness of the core. When preferences are substitutable, our characterization gives a simple proof of the lattice structure of core matchings, and it gives a method for computing the join and meet of two core matchings.
\end{abstract}

JEL classification numbers: $\mathrm{C} 78$

Key words: Matching, Core, Lattice, Stability, Algorithm, Complexity, Substitutability, Tarski's Fixed Point Theorem 


\title{
Core Many-to-one Matchings by Fixed-Point Methods*
}

\author{
Federico Echenique Jorge Oviedo
}

\section{Introduction}

This paper deals with the many-to-one matchings that are in the core. Given a set of workers and a set of firms, a many-to-one matching (a matching, for short) is an assignment of a group of workers to each firm. The problem is interesting because each worker has a preference relation over firms, and each firm has a preference relation over groups of workers. Given a matching, firms who are unhappy with their current group of workers, and workers who are unhappy with their current employers, may recontract in a mutually beneficial way, thus destroying the proposed matching. Arguably, the natural formalization of matchings that are robust to recontracting is the core (see Roth and Sotomayor (1990), RS in the rest of the paper, for definitions).

The standard approach to the core (RS is a classic) is to impose some structure on firms' preferences that both simplifies the core and ensures that it is non-empty; the structure used is to assume that firms' preferences are "substitutable" (see RS, or our Definition 5 below). When preferences are substitutable, a matching is in the core if and only if it is "stable" - a stable matching is robust to recontracting by worker-firm pairs, but not more complicated coalitions. Further, there is an algorithm, the "deferred acceptance algorithm" (DAA) which finds certain distinguished core matchings: the DAA finds a core matching that is better for the firms than all other core matchings, and a core matching that is better for the workers than all other core matchings.

Our approach is different. We characterize the core as the set of fixed points of a map $T$. We do not add structure to the model, so the core (and the fixed points of $T$ ) may be empty. Our characterization is useful because it allows us to:

1. Construct a simple algorithm that consists in iterating $T$, we shall call it the $T$ algorithm. The $T$-algorithm either finds a core matching or cycles in a recognizable way. Unlike the DAA, the $T$-algorithm does not stop at a non-core matching. We present an example where the DAA stops at a non-core matching, while the $T$-algorithm succeeds in finding a core matching.

*We are grateful to Alejandro Neme and Ruth Martínez for their helpful comments. 
2. We prove that, when preferences are substitutable, $T$ has at least one fixed pointhence that the core is non-empty - and that the $T$-algorithm finds the two distinguished core matchings: the matching that is best for the firms and the matching that is best for the workers.

We can bound the computational complexity of the $T$-algorithm. To the best of our knowledge, the computational complexity of the DAA for the many-to-one case is unknown. ${ }^{1}$

3. Blair (1988) proves that, when preferences are substitutable, the core matchings have a lattice structure.

(a) Our characterization gives a very simple proof of Blair's result; it follows almost immediately from Tarski's fixed point theorem, as $T$ is a monotone map under an appropriate order.

(b) There is no formula for computing Blair's lattice operations (join and meet). We show how the $T$-algorithm computes the join and meet of any two core matchings.

Our results on the core build a bridge to the literature on supermodular games (see Topkis (1998)); in fact, our results on the $T$-algorithm are adapted from similar results on computing Nash equilibria in supermodular games. We believe that this bridge is useful for understanding matching problems better, and may deliver further results in future research.

Fixed-point methods have been used in the matching literature before. Tarski's fixedpoint theorem was applied by Roth and Sotomayor (1988) to the assignment game, Adachi (2000) to one-to-one stable matchings, and Fleiner (2001) to many-to-one stable matchings. Fleiner's is the paper closest to our paper; he proves a version of Blair's lattice result for a general graph-theoretic model. Fleiner's model includes the manyto-one matching model with substitutable preferences as a special case. Fleiner's paper is a paper in graph theory, though, and his results are very hard to translate into the language of matching models. For that reason, we believe that our result in (3.a) above is valuable, beyond its auxiliary role in our other results.

We should clarify at this point that "core" is RS's "weak core." We do not have results for RS's "strong core," hence we opted, in the interest of exposition economy, for omitting all "weak" qualifiers from the paper. Nothing is really known about the strong core, so we feel that our omission is justified. See RS, and Sotomayor (1999), for a discussion of the different core definitions.

In Section 2 we define the model, and prove some preliminary results on the core manyto-one matchings. In Section 3 we introduce our fixed-point approach to the core. In Section 4 we introduce the $T$-algorithm. In Section 5 we study the lattice structure of the core many-to-one matchings. In Section 6 we analyze the $T$-algorithm for substitutable preferences.

\footnotetext{
${ }^{1}$ Gusfield and Irving (1989) prove that the DAA is $O\left(n^{2}\right)$ for one-to-one matchings.
} 


\section{Preliminary definitions and results}

\subsection{The Model}

There are two disjoint sets of agents, the set of $n$ firms $F$ and the set of $m$ workers $W$. Each firm $f \in F$ has a strict, transitive, and complete preference relation $P(f)$ over the set of all subsets of $W$, and each worker has a strict, transitive, and complete preference relation $P(w)$ over $F \cup \emptyset$. Preferences profiles are $(n+m)$-tuples of preference relations; we denote them by $P=\left(P\left(f_{1}\right), \ldots, P\left(f_{n}\right) ; P\left(w_{1}\right), \ldots, P\left(w_{m}\right)\right)$.

Given a firm's preference relation, $P(f)$, the sets of workers that $f$ prefers to the empty set are called acceptable; thus we allow that firm $f$ may prefer not hiring any worker rather than hiring unacceptable subsets of workers. Similarly, given a preference relation of a worker $P(w)$ the firms preferred by $w$ to the empty set are called acceptable; in this case we are allowing that worker $w$ may prefer to remain unemployed rather than working for an unacceptable firm. It turns out that only acceptable partners matter, so we shall write preference relation concisely as lists of acceptable partners. For example,

$$
P\left(f_{i}\right)=\left\{w_{1}, w_{3}\right\},\left\{w_{2}\right\},\left\{w_{1}\right\},\left\{w_{3}\right\}
$$

indicates that $\left\{w_{1}, w_{3}\right\} P\left(f_{i}\right)\left\{w_{2}\right\} P\left(f_{i}\right)\left\{w_{1}\right\} P\left(f_{i}\right)\left\{w_{3}\right\} P\left(f_{i}\right) \emptyset$ and

$$
P\left(w_{j}\right)=f_{1}, f_{3}
$$

indicates that $f_{1} P\left(w_{j}\right) f_{3} P\left(w_{j}\right) \emptyset$.

We denote by $R$ the weak orders associated with $P$. So, $f_{i} R(w) f_{j}$ if $f_{i}=f_{j}$ or $f_{i} P(w) f_{j}$. Similarly for $R(f)$.

We shall study assignments of workers to firms as matchings of firms with groups of workers. The assignment problem consists of matching workers with firms maintaining the bilateral nature of their relationship, and allowing for the possibility that both firms and workers may remain unmatched. Formally,

Definition 1 A matching $\mu$ is a mapping from the set $F \cup W$ into the set of all subsets of $F \cup W$ such that for all $w \in W$ and $F \in F$ :

1. Either $|\mu(w)|=1$ and $\mu(w) \subseteq F$ or else $\mu(w)=\emptyset$.

2. $\mu(f) \in 2^{W}$.

3. $\mu(w)=\{f\}$ if and only if $w \in \mu(f) .^{2}$

\footnotetext{
${ }^{2}$ We shall often abuse notation by omitting the brackets to denote a set with a unique element; here we write $\mu(w)=f$ instead of $\mu(w)=\{f\}$.
} 
Given $(F, W, P)$ we denote the set of matchings by $\mathcal{M}$. A matching $\mu$ is said to be one-to-one if firms can hire at most one worker; namely, condition 2 is replaced by: Either $|\mu(f)|=1$ and $\mu(f) \subseteq W$ or else $\mu(f)=\emptyset$. The model in which all matchings are one-to-one is also known in the literature as the marriage model.

We shall represent matchings as follows. Let $F=\left\{f_{1}, f_{2}, f_{3}\right\}$ be the set of firms, and $W=\left\{w_{1}, w_{2}, w_{3}, w_{4}\right\}$ the set of workers. One possible matching is:

$$
\mu=\left\{\begin{array}{cccc}
f_{1} & f_{2} & f_{3} & \emptyset \\
\left.w_{3}, w_{4}\right\} & \left\{w_{1}\right\} & \emptyset & \left\{w_{2}\right\}
\end{array}\right.
$$

where firm $f_{1}$ is matched to workers $w_{3}$ and $w_{4}$, firm $f_{2}$ is matched to worker $w_{1}$, and firm $f_{3}$ and worker $w_{2}$ are unmatched.

Let $P$ be a preference profile. Given a set $S \subseteq W$, let $C h(S, P(f))$ denote firm $F$ 's most-preferred subset of $S$ according to its preference ordering $P(f)$. We call $C h(S, P(f))$ the choice set of $\mathrm{S}$ according to $P(f)$. That is, $A=C h(S, P(f))$ if and only if $A \subseteq S$ and $A P(f) B$ for all $B \subseteq S$ with $A \neq B$.

\subsection{The core, stable matchings, and stable* matchings}

A matching $\mu$ is stable if no worker-firm pair can benefit by deviating from $\mu$. RS prove that, if firms' preferences are substitutable, the set of stable matchings equals the core.

A matching is stable* if no set of workers can, jointly with one firm, benefit from deviating from $\mu$. We prove that the set of stable* matchings equals the core; the result does not require additional assumptions on preferences. We use the notion of stable* matching instead of stable matching because our fixed-point approach allows us to characterize the set of stable* matchings, and thus the core. It should be clear from our proofs and examples just how useful the stable* definition is.

Let $P$ be a preference profile and let $\mu$ be a matching. Say that $\mu$ is individually rational if $\mu(w) R(w) \emptyset$ for all $w \in W$, and if $\mu(f)=C h(\mu(f), P(f))$, for all $f \in$ $F$. Intuitively, $\mu$ is individually rational if no agent can unilaterally improve over its assignment by $\mu$-workers by choosing to remain unemployed and firms by firing some of its workers.

A worker-firm pair $(w, f)$ blocks $\mu$ if $w \notin \mu(f), w \in C h(\mu(f) \cup\{w\}, P(f))$, and $f P(w) \mu(w)$; that is, if $w$ and $f$ are not matched through $\mu$, firm $f$ wants to hire $w-$ possibly after firing some of its current workers in $\mu(f)$ - and worker $w$ prefers firm $f$ over her current match $\mu(w)$.

Let $P$ be a preference profile and let $\mu$ be a matching. A pair $(B, f) \in 2^{W} \times F$, with $B \neq \emptyset$, blocks $^{*} \mu$ if

$$
f P(w) \mu(w),
$$


for all $w \in B$, and there is $A \subseteq \mu(f)$ such that

$$
[A \cup B] P(f) \mu(f) .
$$

In words, $(B, f)$ blocks* $^{*}$ if firm $f$ is willing to hire the workers in $B$-possibly after firing some of its current workers in $\mu(f)$-and all workers $w$ in $B$ prefer $f$ over their current match $\mu(w)$.

Definition 2 A matching $\mu$ is stable if is individually rational and there is no workerfirm pair that blocks $\mu$. A matching $\mu$ is stable* if it is individually rational and there is no pair that blocks* $\mu$.

Notation. Given a preference profile $P$, we denote the set of stable matchings by $S(P)$, and the set of stable* matchings by $S^{*}(P)$.

Remark. We shall show below that $S^{*}(P)$ equals the core. It follows then from results in RS that $S^{*}(P) \subseteq S(P)$ and that, in general, $S^{*}(P) \neq S(P)$. As stable* is a new concept, we include independent proofs of these two facts in Lemma 1 and Example 2.

Lemma $1 S^{*}(P) \subseteq S(P)$

Proof. Let $\mu \in S^{*}(P)$, and assume that $\mu \notin S(P)$. Since $\mu$ is individually rational, and $\mu \notin S(P)$, there exist $(w, f) \in W \times F$ such that

$$
f P(w) \mu(w)
$$

and

$$
w \in C h(\mu(f) \cup\{w\}, P(f)) .
$$

Statement (2) implies that

$$
C h(\mu(f) \cup\{w\}, P(f)) P(f) \mu(f) .
$$

Let $B=\{w\}$, and $A=C h(\mu(f) \cup\{w\}, P(f)) \cap \mu(f)$. We shall prove that $(B, f)$ blocks* $\mu$. Since $B=\{w\}$, statement (1) gives us the first part of the definition of block*. Also,

$$
\begin{aligned}
A & =C h(\mu(f) \cup\{w\}, P(f)) \cap \mu(f) \\
& =C h(\mu(f) \cup\{w\}, P(f)) \backslash\{w\} \\
& =C h(\mu(f) \cup\{w\}, P(f)) \backslash B,
\end{aligned}
$$

so statement (3) implies that

$$
[A \cup B]=C h(\mu(f) \cup\{w\}, P(f)) P(f) \mu(f),
$$

and we have the second part of the definition of block* . Thus, $\mu \notin S^{*}(P)$. 
Example 2 This example shows that $S^{*}(P)$ and $S(P)$ may be different. Let $F=\{f\}$ and $W=\left\{w_{1}, w_{2}, w_{3}\right\}$ be the sets of agents. Let the preference profile $P$ be defined as:

$$
\begin{aligned}
& P(f)=\left\{w_{1}, w_{2}\right\},\left\{w_{3}\right\} \\
& P\left(w_{1}\right)=f \\
& P\left(w_{2}\right)=f, \text { and } \\
& P\left(w_{3}\right)=f .
\end{aligned}
$$

Consider the following matchings:

$$
\begin{array}{cccccc}
f & \emptyset & & f & \emptyset & \emptyset \\
\mu_{1}= & \left\{w_{1}, w_{2}\right\} & \left\{w_{3}\right\}
\end{array} \text { and } \quad \mu_{2}=\begin{array}{ccc}
\left\{w_{3}\right\} & \left\{w_{1}\right\} & \left\{w_{2}\right\}
\end{array} .
$$

We have that $\mu_{2} \notin S^{*}(P)$, because there exists $\left(\left\{w_{1}, w_{2}\right\}, f\right)$ such that

$$
\begin{aligned}
& f P\left(w_{1}\right) \mu_{1}\left(w_{1}\right)=\emptyset \\
& f P\left(w_{2}\right) \mu_{1}\left(w_{2}\right)=\emptyset
\end{aligned}
$$

and $\left(A=\emptyset \subseteq \mu_{2}(f)\right)$

$$
\left\{w_{1}, w_{2}\right\} P(f) \mu_{2}(f)=\left\{w_{3}\right\} .
$$

Also we have that $S(P)=\left\{\mu_{1}, \mu_{2}\right\}$, and $S^{*}(P)=\left\{\mu_{1}\right\}$.

Definition 3 Let $P$ be a preference profile. The core is the set of matchings $\mu$ for which there is no $F^{\prime} \subseteq F, W^{\prime} \subseteq W$ with $F^{\prime} \cup W^{\prime} \neq \emptyset$, and $\hat{\mu} \in \mathcal{M}$ such that: for all $w \in W^{\prime}$, and for all $f \in F^{\prime}$

1. $\hat{\mu}(w) \subseteq F^{\prime}$, and $\hat{\mu}(f) \subseteq W^{\prime}$,

2. $\hat{\mu}(w) R(w) \mu(w)$,

3. $\hat{\mu}(f) R(f) \mu(f)$,

4. and $\hat{\mu}(s) P(s) \mu(s)$ for at least one $s \in W^{\prime} \cup F^{\prime}$.

We shall denote the core by $C_{w}(P)$.

Theorem $3 S^{*}(P)=C_{w}(P)$.

Proof. First we shall prove that $S^{*}(P) \subseteq C_{w}(P)$. Let $\mu \in S^{*}(P)$, and suppose that $\mu \notin C_{w}(P)$. Let $F^{\prime} \subseteq F, W^{\prime} \subseteq W$ with $F^{\prime} \cup W^{\prime} \neq \emptyset$, and let $\hat{\mu} \in \mathcal{M}$ such that, for all $w \in W^{\prime}$, and for all $f \in F^{\prime}$

$$
\begin{gathered}
\hat{\mu}(w) \subseteq F^{\prime}, \text { and } \hat{\mu}(f) \subseteq W^{\prime}, \\
\hat{\mu}(w) R(w) \mu(w), \\
\hat{\mu}(f) R(f) \mu(f),
\end{gathered}
$$


and

$$
\hat{\mu}(s) P(s) \mu(s) \text { for at least one } s \in W^{\prime} \cup F^{\prime} .
$$

We shall need the following

Claim. There exists $f \in F^{\prime}$, such that $\hat{\mu}(f) P(f) \mu(f)$ if and only if there is $w \in W^{\prime}$ such that $\hat{\mu}(w) P(w) \mu(w)$.

Proof of The ClAim. Let $\hat{\mu}(f) P(f) \mu(f)$. Because $\mu$ is individually rational, we have that $\hat{\mu}(f) \nsubseteq \mu(f)$, so let $\bar{w} \in \hat{\mu}(f) \backslash \mu(f)$. By condition (4), we have that $\bar{w} \in \hat{\mu}(f) \subseteq W^{\prime}$; but then $\bar{w} \notin \mu(f)$ and condition (5) implies that

$$
\hat{\mu}(\bar{w}) P(\bar{w}) \mu(\bar{w}) .
$$

Now suppose there exists $w \in W^{\prime}$ such that $\hat{\mu}(w) P(w) \mu(w)$. Let $\bar{f}=\hat{\mu}(w)$. Then $\bar{f} \neq$ $\mu(w)$, so $w \notin \mu(\bar{f})$. Thus $\hat{\mu}(\bar{f}) \neq \mu(\bar{f})$, and condition $(6)$ implies that $\hat{\mu}(\bar{f}) P(\bar{f}) \mu(\bar{f})$. This proves the claim.

By the claim, we can assume that there exists $f \in F^{\prime}$ such that $\hat{\mu}(f) \neq \mu(f)$. Let $B=\hat{\mu}(f) \backslash \mu(f)$ and $A=\hat{\mu}(f) \cap \mu(f)$ then

$$
A \cup B=\hat{\mu}(f) P(f) \mu(f) .
$$

Now, $B \subseteq \hat{\mu}(f) \subseteq W^{\prime}$, and $B \cap \mu(f)=\emptyset$. Condition (5) implies that, for all $w \in B$,

$$
f=\hat{\mu}(w) P(w) \mu(w) .
$$

Statements (7) and (8) contradict that $\mu \in S^{*}(P)$.

Now we shall prove that $C_{w}(P) \subseteq S^{*}(P)$. Let $\mu \in C_{w}(P)$, and suppose that $\mu \notin$ $S^{*}(P)$. First we shall show that $\mu$ is individually rational. Assume that there exists $f \in F$ such that

$$
\mu(f) \neq C h(\mu(f), P(f)) .
$$

Let $\widehat{\mu}(f)=C h(\mu(f), P(f)), F^{\prime}=\{f\} \subseteq F, W^{\prime}=\widehat{\mu}(f) \subseteq W$. It is easy to see that $\mu \notin C_{w}(P)$, as $\hat{\mu}(w) R(w) \mu(w)$ for all $w \in W^{\prime}$, while $\hat{\mu}(f) R(f) \mu(f)$. Thus,

$$
\mu(f)=C h(\mu(f), P(f)),
$$

for all $f \in F$.

Now suppose there is $w \in W$ such that

$$
\emptyset P(w) \mu(w)
$$

Let $\widehat{\mu}(w)=\emptyset, W^{\prime}=\{w\}, F^{\prime}=\emptyset$. As before, $\mu \notin C_{w}(P)$ because $\hat{\mu}(w) P(w) \mu(w)$. Thus,

$$
\mu(w) R(w) \emptyset
$$


for all $w \in W$. Statements (9) and (10) establish that $\mu$ is individual rational.

Assume that there exist $(B, f) \in 2^{W} \times F$ with $B \neq \emptyset$, such that: for all $w \in B$

$$
f P(w) \mu(w)
$$

and there exists $A \subseteq \mu(f)$ such that

$$
[A \cup B] P(f) \mu(f) .
$$

Letting $\hat{\mu}(f)=A \cup B, F^{\prime}=\{f\}$ and $W^{\prime}=B$, it follows that $\mu \notin C_{w}(P)$. A contradiction.

\subsection{Lattices and chains}

Let $X$ be a set, and $R$ a partial order - a transitive, reflexive, antisymmetric binary relation-on $X$. For any subset $A$ of $X, \inf _{R} A\left(\sup _{R} A\right)$ is the greatest (lowest) lower (upper) bound on $A$, in the order $R$. We say that the pair $(X, R)$ is a lattice if whenever $x, y \in X$, both $x \wedge_{R} y=\inf _{R}\{x, y\}$ and $x \vee_{R} y=\sup _{R}\{x, y\}$ exist in $X$. We say that a lattice $(X, R)$ is complete if for every nonempty subset $A$ of $X, \inf _{R} A$ and $\sup _{R} A$ exist in $X$. Note that any finite lattice is complete. A lattice $(X, R)$ is a chain if, for any $x, y \in X, x R y$ or $y R x$, or both, are true.

\section{The core as a set of fixed points}

We shall construct a map $T$ on a superset of $\mathcal{M}$ such that the set of fixed points of $T$ is the core.

\subsection{Pre-matchings}

Say that a pair $\nu=\left(\nu_{F}, \nu_{W}\right)$, with $\nu_{F}: F \rightarrow 2^{W}$ and $\nu_{W}: W \rightarrow F \cup\{\emptyset\}$, is a prematching. Let $\mathcal{V}_{W}\left(\mathcal{V}_{F}\right)$ denote the set of all $\nu_{W}\left(\nu_{F}\right)$ functions. Thus, $\mathcal{V}=\mathcal{V}_{F} \times \mathcal{V}_{W}$ denotes the set of pre-matchings $\nu=\left(\nu_{F}, \nu_{W}\right)$. The superset of $\mathcal{M}$ we need turns out to be the set of pre-matchings $\mathcal{V}$ (pre-matchings were first used by Adachi (2000)).

A pre-matching $\nu=\left(\nu_{F}, \nu_{W}\right)$, and a matching $\mu$ are equivalent if $\mu(f)=\nu_{F}(f)$ for all $f$, and $\mu(w)=\nu_{W}(w)$ for all $w$. A pre-matching $\nu=\left(\nu_{F}, \nu_{W}\right)$ is equivalent to some matching if and only if $\nu$ is such that $\nu_{W}(w)=f$ if and only if $w \in \nu_{F}(f)$.

\section{Notation.}


1. We shall identify matchings and pre-matchings when they are equivalent, so if $\nu \in \mathcal{V}$ is equivalent to some matching $\mu$ we shall say that $\nu$ is a matching. Hence $\mathcal{M}$ is a subset of $\mathcal{V}$.

2. If $\nu=\left(\nu_{F}, \nu_{W}\right)$ is a pre-matching, we shall some times write $\nu(f)$ for $\nu_{F}(f)$ and $\nu(w)$ for $\nu_{W}(w)$.

\subsection{T and the core}

Let $\nu$ be a pre-matching, and let

$$
U(f, \nu)=\{w \in W: f R(w) \nu(w)\}
$$

and

$$
V(w, \nu)=\{f \in F: w \in C h(\nu(f) \cup\{w\}), P(f)\} \cup\{\emptyset\} .
$$

The set $U(f, \nu)$ is the set of workers $w$ that are willing to give up $\nu(w)$ in exchange for $f$. The set $V(w, \mu)$ is the set of firms $f$ that are willing to hire $w$, possibly after firing some of the workers it was assigned by $\nu$.

Now, define $T: \mathcal{V} \rightarrow \mathcal{V}$ by

$$
(T \nu)(s)= \begin{cases}C h(U(s, \nu), P(s)) & \text { if } s \in F \\ \max _{P(s)}\{V(s, \nu)\} & \text { if } s \in W .\end{cases}
$$

The map $T$ has a simple interpretation: $(T \nu)(f)$ is firm f's optimal team of workers, among those willing to work for $f$, and $(T \nu)(w)$ is the firm preferred by $w$ among the firms that are willing to hire $w$.

Notation. We shall denote by $\mathcal{E}$ the set of fixed points of $T$, so $\mathcal{E}=\{\nu \in \mathcal{V}: \nu=T \nu\}$.

Theorem $4 \mathcal{E}=S^{*}(P)$.

Before we prove Theorem 4, note that Theorems 3 and 4 imply:

Corollary $5 \mathcal{E}=C_{w}(P)$.

Proof of Theorem 4 . To make the proof clearer, we present the first part of the proof as Lemma 6.

Lemma 6 If $\nu \in \mathcal{E}$ then $\nu$ is a matching and $\nu$ is individually rational.

Proof. Let $\nu=\left(\nu_{F}, \nu_{W}\right) \in \mathcal{E}$. 
1. Fix $w \in \nu_{F}(f)$, we shall prove that $f=\nu_{W}(w) . \nu \in \mathcal{E}$ implies that

$$
w \in \nu_{F}(f)=(T \nu)(f)=C h(U(f, \nu), P(f)) .
$$

Thus $w \in U(f, \nu)$.

The definition of $U(f, \nu)$ implies

$$
f R(w) \nu_{W}(w) .
$$

Now, $\nu_{F}(f) \cup\{w\}=\nu_{F}(f)$ and $\nu \in \mathcal{E}$, imply that

$$
\nu_{F}(f)=(T \nu)(f)=C h(U(f, \nu), P(f)) .
$$

So

$$
\begin{aligned}
C h\left(\nu_{F}(f), P(f)\right) & \stackrel{(1)}{=} C h(C h(U(f, \nu), P(f)), P(f)) \\
& \stackrel{(2)}{=} C h(U(f, \nu), P(f)) \\
& \stackrel{(3)}{=} \nu_{F}(f) .
\end{aligned}
$$

Equalities (1) and (3) follow from statement (12). Equality (2) is a simple property of choice sets: $C h(C h(S, P(f)), P(f))=C h(S, P(f))$. Hence we have that

$$
\nu_{F}(f)=C h\left(\nu_{F}(f), P(f)\right) .
$$

Now $w \in \nu_{F}(f)$ implies that $C h\left(\nu_{F}(f), P(f)\right)=C h\left(\nu_{F}(f) \cup\{w\}, P(f)\right)$. So statement (13) implies that $f \in V(w, \nu)$. But

$$
\nu_{W}(w)=(T \nu)(w)=\max _{P(w)}\{V(w, \nu)\},
$$

so

$$
\nu_{W}(w) R(w) f .
$$

Statements (11), (14) and anti-symmetry of preference relations imply that, $f=$ $\nu_{W}(w)$.

2. Let $f=\nu_{W}(w)$, we shall prove that $w \in \nu_{F}(f)$. First, note that $f=\nu_{W}(w)$ implies that

$$
w \in U(f, \nu) .
$$

Second, note that

$$
f=\nu_{W}(w)=(T \nu)(w)=\max _{P(w)}\{V(w, \nu)\},
$$

because $\nu \in \mathcal{E}$. Let us then record here

$$
\nu_{W}(w) R(w) \emptyset .
$$

Now, $f \in V(w, \nu)$, so we must have $w \in C h\left(\nu_{F}(f) \cup\{w\}, P(f)\right)$ by the definition of $V(w, \nu)$. That is,

$$
w \in C h\left(\nu_{F}(f) \cup\{w\}, P(f)\right) R(f) \nu_{F}(f),
$$


Now, $\nu \in \mathcal{E}$ implies that

$$
\nu_{F}(f)=(T \nu)(f)=C h(U(f, \nu), P(f)) .
$$

The definition of choice set implies

$$
\nu_{F}(f) \subseteq U(f, \nu),
$$

and

$$
\nu_{F}(f) R(f) U(f, \nu) .
$$

But statements (15) and (19) give

$$
U(f, \nu) \supseteq C h\left(\nu_{F}(f) \cup\{w\}, P(f)\right) .
$$

The definition of choice set implies

$$
\nu_{F}(f) R(f) C h\left(\nu_{F}(f) \cup\{w\}, P(f)\right) .
$$

Statements (17) and (20), and anti-symmetry of the preference relations imply that, $w \in \nu_{F}(f)$.

By 1 and 2, $w \in v_{F}(f)$ if and only if $f=v_{W}(w)$. So, $\nu$ is a matching. Statements (13) and (16) imply that $\nu$ is individually rational.

Now we finish the proof of Theorem 4

Let $\mu \in \mathcal{E}$. By Lemma 6 we know that $\mu$ is an individually rational matching.

Fix $f \in F, B \subseteq W$ such that $B \neq \emptyset$. We assume that, for all $w \in B$,

$$
f P(w) \mu(w) .
$$

By the definition of $U(f, \mu)$, we have that

$$
B \subseteq U(f, \mu) .
$$

Let $A \subseteq \mu(f)$. Because $\mu$ is a matching, we have that for all $w \in A, f=\mu(w)$. So the definition of $U(f, \mu)$ implies that

$$
A \subseteq U(f, \mu)
$$

Now, $\mu \in \mathcal{E}$ so $\mu(f)=(T \mu)(f)=C h(U(f, \mu), P(f))$; statements (22) and (23) imply then

$$
\mu(f) R(f) C h(A \cup B, P(f)) R(f) A \cup B .
$$

Statements $(21)$ and $(24)$ show that there is not $(B, f)$ that blocks* $\mu$. Thus $\mu \in S^{*}(P)$.

Let $\mu \in S^{*}(P)$ and assume that $\mu \neq T \mu$. First assume that there exist $f \in F$ such that

$$
\mu(f) \neq(T \mu)(f)=C h(U(f, \mu), P(f))=C \subseteq U(f, \mu) .
$$


Let $A=C \cap \mu(f)$, and $B=C \backslash \mu(f)$. Because $\mu$ is an individually rational matching we have that $\mu(f) \subseteq U(f, \mu)$ and $B \neq \emptyset$. Now,

$$
A \cup B=C P(f) \mu(f) .
$$

Also,

$$
f P(w) \mu(w)
$$

for all $w \in B$, as $B \subseteq C \subseteq U(f, \mu)$. Statements (25) and (26) imply that $(B, f)$ block* $^{*}$ $\mu$, which contradicts that $\mu \in S^{*}(P)$.

Hence, for all $f \in F$,

$$
\mu(f)=(T \mu)(f)
$$

Assume that there exists $w \in W$ such that

$$
\mu(w) \neq(T \mu)(w)=\max _{P(w)}\{V(w, \mu)\}=\bar{f} \in V(w, \mu)
$$

SO

$$
w \in C h(\mu(\bar{f}) \cup\{w\}, P(\bar{f})),
$$

by the definition on $V(w, \mu)$.

But $\mu$ is a matching, so we have that $w \notin \mu(\bar{f})$ and $\mu(w) \in V(w, \mu)$. This implies that

$$
\bar{f} P(w) \mu(w)
$$

and

$$
w \in C h(\mu(f) \cup\{w\}, P(f))=C .
$$

Let $A=C \cap \mu(f)=C-\{w\}$, and $B=\{w\}$. Then $(B, f)$ blocks* $\mu$ because of

$$
C=A \cup B P(f) \mu(f),
$$

and statement (28). Hence, for all $w \in W$,

$$
\mu(w)=(T \mu)(w)
$$

Statements (27) and (29) imply that $\mu=T \mu$. Hence $\mu \in \mathcal{E}$.

\section{The $T$-algorithm}

The $T$-algorithm is very simple: start at some $\nu \in \mathcal{V}$ and iterate $T \nu$ until two iterations are identical. When two iterations are identical, stop.

We prove that, when the $T$-algorithm stops, it must be at a core matching. Further, we show that once the $T$-algorithm hits on a matching $\mu$ (not just a pre-matching), $\mu$ must be in the core. Hence, the $T$-algorithm "wanders" around pre-matchings until it 
finds a matching - it will stop at the matching it finds, and it will be a core matching. It follows, of course, that if the core is empty the algorithm must cycle, and that the cycle will only involve pre-matchings that are not matchings.

The algorithm is very simple, but we include a detailed description to be as clear as possible, and to introduce some notation that we shall use in the proofs below:

\section{Algorithm 1 (T-algorithm)}

1. Set $\nu^{0}=\nu$. Set $\nu^{1}=T \nu^{0}$ and $k=1$.

2. While $\nu^{k} \neq \nu^{k-1}$, do:

(a) Set $k=k+1$,

(b) $\operatorname{set} \nu^{k}=T \nu^{k-1}$.

3. Set $\mu=\nu^{k}$. Stop.

Proposition 7 If the $T$-algorithm stops at $\mu \in \mathcal{V}$, then $\mu$ is a matching $(\mu \in \mathcal{M})$ and $\mu$ is in the core. If $\nu^{k}$ is in the core, for some iteration $k$ of the T-algorithm, then the algorithm stops at $\mu=\nu^{k}$.

Proof. If the algorithm stops at $\mu$ then $\nu^{k}=\nu^{k-1}=\mu$. Then, $\mu=T \nu^{k-1}=T \mu$, so $\mu \in \mathcal{E}$. By Corollary $5, \mu \in C_{w}(P)$. On the other hand, if $\nu^{k}$ is in the core, then $\nu^{k}$ is a fixed point of $T$ by Corollary 5 . Then the $T$-algorithm stops at $\mu=\nu^{k}$.

Proposition 8 If $\nu^{k}$ is a matching, for some iteration $k$ in the T-algorithm, then the algorithm stops at $\mu=\nu^{k}$, and thus $\nu^{k}$ is a core matching.

We shall present the proof of Proposition 8 as two lemmata:

Lemma 9 Let $\nu \in \mathcal{V}$ be a pre-matching. If $\nu^{1}=T \nu$ is a matching, then $\nu^{1}$ is individually rational.

Proof. For all $f \in F$,

$$
\nu^{1}(f)=(T \nu)(f)=C h(U(f, \nu), P(f)) .
$$

So

$$
\begin{aligned}
C h\left(\nu^{1}(f), P(f)\right) & \stackrel{(1)}{=} C h(C h(U(f, \nu), P(f)), P(f)) \\
& \stackrel{(2)}{=} C h(U(f, \nu), P(f)) \\
& \stackrel{(3)}{=} \nu^{1}(f) .
\end{aligned}
$$

Equalities (1) and (3) follow because $\nu^{1}=T \nu$. Equality (2) is a property of choice sets. So, for all $f \in F$,

$$
\nu^{1}(f)=C h\left(\nu^{1}(f), P(f)\right)
$$


For all $w \in W$,

$$
\nu^{1}(w)=(T \nu)(w)=\max _{P(w)}\{V(w, \nu)\} \cup\{\emptyset\}
$$

So,

$$
\nu^{1}(w) R(w) \emptyset .
$$

Statements (30) and (31) show that $\nu^{1}$ is individually rational.

Lemma 10 If $T \nu$ is a matching, for some $\nu \in \mathcal{V}$, then $T \nu$ is a core matching. Thus if $\nu^{k}=T \nu^{k-1}$ is a matching, then the T-algorithm must stop at $\nu^{k}$, and $\nu^{k}$ is a core matching.

Proof. We shall prove that, if $\nu \in \mathcal{V}$ is a pre-matching, and $\nu^{1}=T \nu$ is a matching, then $\nu^{1} \in S^{*}(P)$. The lemma follows from Proposition 7, as $S^{*}(P)=\mathcal{E}=C_{w}(P)$.

Assume that $\nu^{1} \notin S^{*}(P)$. By Lemma $9, \nu^{1}$ is individually rational, so there must exist a $(B, f)$ that blocks $\nu^{1}$. Hence, for all $w \in B(B \neq \emptyset)$,

$$
f P(w) \nu^{1}(w),
$$

and there must exist $A \subseteq \nu^{1}(f)$ such that

$$
[A \cup B] P(f) \nu^{1}(f) .
$$

But $\nu^{1}$ is a matching, and $\nu^{1}=T \nu$, so we have that for all $w \in B, w \in \nu^{1}\left(\nu^{1}(w)\right)$. But $\nu^{1}\left(\nu^{1}(w)\right)=(T \nu)\left(\nu^{1}(w)\right)=C h\left(U\left(\nu^{1}(w), \nu\right), P\left(\nu^{1}(w)\right)\right)$, so that, $w \in U\left(\nu^{1}(w), \nu\right)$, i.e.

$$
\nu^{1}(w) R(w) \nu(w) .
$$

Statements (32) and (34) imply that, for all $w \in B, f R(w) \nu(w)$, i.e.

$$
B \subseteq U(f, \nu) .
$$

Now, $A \subseteq \nu^{1}(f)$ and $\nu^{1}(f)=(T \nu)(f)=C h(U(f, \nu), P(f))$, so

$$
A \subseteq U(f, \nu) .
$$

Statements (33), (35), and (36) contradict that $\nu^{1}(f)=(T \nu)(f)=C h(U(f, \nu), P(f))$. Thus $\nu^{1} \in S^{*}(P)$.

\section{Remarks.}

- Proposition 8, and Example 11 below, imply that the $T$-algorithm and the DAA are different algorithms; we have not simply written down the DAA using different language. 
- Unlike the DAA, the T-algorithm only stops at a core matchings. Example 11 below shows how the $T$-algorithm succeeds in finding a core matching, while the DAA stops at a non-core matching.

- When the core is empty, the T-algorithm must cycle. We illustrate this in Example 12 below.

Example 11 Let $F=\left\{f_{1}, f_{2}\right\}, W=\left\{w_{1}, w_{2}, w_{3}, w_{4}\right\}$, and the preference profile $P$ be defined by:

$$
\begin{aligned}
& P\left(f_{1}\right)=\left\{w_{1}, w_{2}\right\},\left\{w_{3}, w_{4}\right\},\left\{w_{1}, w_{3}\right\},\left\{w_{2}, w_{4}\right\},\left\{w_{1}\right\},\left\{w_{2}\right\},\left\{w_{3}\right\},\left\{w_{4}\right\} \\
& P\left(f_{2}\right)=\left\{w_{1}, w_{2}\right\},\left\{w_{1}, w_{3}\right\},\left\{w_{2}, w_{4}\right\},\left\{w_{3}, w_{4}\right\},\left\{w_{1}\right\},\left\{w_{2}\right\},\left\{w_{3}\right\},\left\{w_{4}\right\} \\
& P\left(w_{1}\right)=f_{1}, f_{2}, \\
& P\left(w_{2}\right)=f_{2}, f_{1}, \\
& P\left(w_{3}\right)=f_{1}, f_{2}, \text { and } \\
& P\left(w_{4}\right)=f_{1}, f_{2} .
\end{aligned}
$$

Note that $P$ is not substitutable, as $w_{1} \in C h\left(\left\{w_{1}, w_{2}, w_{3}, w_{4}\right\}, P\left(f_{1}\right)\right)=\left\{w_{1}, w_{2}\right\}$ while $w_{1} \notin C h\left(\left\{w_{1}, w_{3}, w_{4}\right\}, P\left(f_{1}\right)\right)=\left\{w_{3}, w_{3}\right\}$. The T-algorithm starting at

$$
\nu^{0}=\begin{array}{cccccc}
f_{1} & f_{2} & w_{1} & w_{2} & w_{3} & w_{4} \\
\left.w_{1}, w_{2}\right\} & \left\{w_{1}, w_{2}\right\} & \emptyset & \emptyset & \emptyset & \emptyset
\end{array}
$$

does:

$$
\begin{array}{rccccccc}
f_{1} & f_{2} & w_{1} & w_{2} & w_{3} & w_{4} \\
U\left(f, \nu^{0}\right) / V\left(w, \nu^{0}\right) & = & \left\{w_{1}, w_{2}, w_{3}, w_{4}\right\} & \left\{w_{1}, w_{2}, w_{3}, w_{4}\right\} & f_{1}, f_{2} & f_{1}, f_{2} & \emptyset & \emptyset \\
\nu^{1} & = & \left\{w_{1}, w_{2}\right\} & \left\{w_{1}, w_{2}\right\} & f_{1} & f_{2} & \emptyset & \emptyset \\
U\left(f, \nu^{1}\right) / V\left(w, \nu^{1}\right) & = & \left\{w_{1}, w_{3}, w_{4}\right\} & \left\{w_{2}, w_{3}, w_{4}\right\} & f_{1}, f_{2} & f_{1}, f_{2} & \emptyset & \emptyset \\
\nu^{2} & = & \left\{w_{3}, w_{4}\right\} & \left\{w_{2}, w_{4}\right\} & f_{1} & f_{2} & \emptyset & \emptyset \\
U\left(f, \nu^{2}\right) / V\left(w, \nu^{2}\right) & = & \left\{w_{1}, w_{3}, w_{4}\right\} & \left\{w_{2}, w_{3}, w_{4}\right\} & f_{2} & f_{2} & f_{1} & f_{1}, f_{2} \\
\nu^{3} & = & \left\{w_{3}, w_{4}\right\} & \left\{w_{2}, w_{4}\right\} & f_{2} & f_{2} & f_{1} & f_{1} \\
U\left(f, \nu^{3}\right) / V\left(w, \nu^{3}\right) & = & \left\{w_{1}, w_{3}, w_{4}\right\} & \left\{w_{1}, w_{2}\right\} & f_{2} & f_{2} & f_{1} & f_{1}, f_{2} \\
\nu^{4} & = & \left\{w_{3}, w_{4}\right\} & \left\{w_{1}, w_{2}\right\} & f_{2} & f_{2} & f_{1} & f_{1}
\end{array}
$$

Note that $\nu^{i}$ for $i=0,1,2,3$ are not matchings; $\nu^{4}$ is a matching so, by Proposition 8, $\nu^{4}$ is a core matching.

On the other hand, the DAA stops at a matching that is not in the core. For the $D A A$, the equivalent to starting the T-algorithm at $\nu^{0}$ is, as we shall see in Section 5, the DAA with firms proposing. The DAA with firms proposing stops at the matching

$$
\mu=\left\{\begin{array}{cccccc}
f_{1} & f_{2} & w_{1} & w_{2} & w_{3} & w_{4} \\
\left.w_{3}, w_{4}\right\} & \left\{w_{2}\right\} & \emptyset & f_{2} & f_{1} & f_{1} .
\end{array}\right.
$$

But $\mu$ is not in the core, as $f_{2} P\left(w_{1}\right) \mu\left(w_{1}\right)=\emptyset, f_{2} R\left(w_{2}\right) \mu\left(w_{2}\right)=f_{2}$, and $\left\{w_{1}, w_{2}\right\} P\left(f_{2}\right)\left\{w_{2}\right\}$ implies that $\left(\left\{w_{1}, w_{2}\right\}, f_{2}\right)$ blocks* $\mu$. 
Example 12 Now we illustrate the T-algorithm in an example with an empty core. Let $F=\left\{f_{1}, f_{2}, f_{3}\right\}$, and $W=\left\{w_{1}, w_{2}, w_{3}\right\}$. be the sets of agents. Let preferences be:

$$
\begin{aligned}
& P\left(f_{1}\right)=\left\{w_{1}, w_{2}\right\},\left\{w_{3}\right\} \\
& P\left(f_{2}\right)=\left\{w_{2}, w_{3}\right\},\left\{w_{1}\right\} \\
& P\left(f_{3}\right)=\left\{w_{1}, w_{3}\right\},\left\{w_{2}\right\} \\
& P\left(w_{1}\right)=f_{1}, f_{3}, f_{2}, \\
& P\left(w_{2}\right)=f_{2}, f_{1}, f_{3}, \text { and } \\
& P\left(w_{3}\right)=f_{3}, f_{2}, f_{1} .
\end{aligned}
$$

Let us start the T-algorithm at

$$
\nu^{0}=\begin{array}{cccccc}
f_{1} & f_{2} & f_{3} & w_{1} & w_{2} & w_{3} \\
\emptyset & \emptyset & \emptyset & f_{1} & f_{2} & f_{3}
\end{array}
$$

The T-algorithm does

$$
\begin{aligned}
& U\left(f, \nu^{0}\right) / V\left(w, \nu^{0}\right)=\quad \begin{array}{cccccc}
f_{1} & f_{2} & f_{3} & w_{1} & w_{2} & w_{3} \\
\left\{w_{1}\right\} & \left\{w_{2}\right\} & \left\{w_{3}\right\} & f_{2} & f_{3} & f_{1}
\end{array}
\end{aligned}
$$

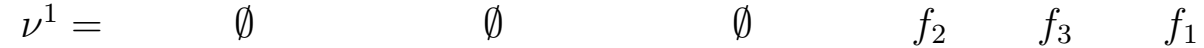

$$
\begin{aligned}
& U\left(f, \nu^{1}\right) / V\left(w, \nu^{1}\right)=\left\{w_{1}, w_{2}, w_{3}\right\} \quad\left\{w_{1}, w_{2}, w_{3}\right\} \quad\left\{w_{1}, w_{2}, w_{3}\right\} \quad f_{2} \quad f_{3} \quad f_{1} \\
& \nu^{2}=\left\{\begin{array}{llllll}
\left\{w_{1}, w_{2}\right\} & \left\{w_{2}, w_{3}\right\} & \left\{w_{1}, w_{3}\right\} & f_{2} & f_{3} & f_{1}
\end{array}\right. \\
& U\left(f, \nu^{2}\right) / V\left(w, \nu^{2}\right)=\left\{w_{1}, w_{2}, w_{3}\right\} \quad\left\{w_{1}, w_{2}, w_{3}\right\} \quad\left\{w_{1}, w_{2}, w_{3}\right\} \quad f_{1}, f_{3} \quad f_{1}, f_{2} \quad f_{2}, f_{3}
\end{aligned}
$$

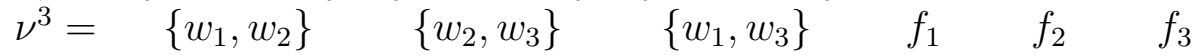

$$
\begin{aligned}
& U\left(f, \nu^{3}\right) / V\left(w, \nu^{3}\right)=\quad\left\{w_{1}\right\} \quad\left\{w_{2}\right\} \quad\left\{w_{3}\right\} \quad f_{1}, f_{3} f_{1}, f_{2} f_{2}, f_{3}
\end{aligned}
$$

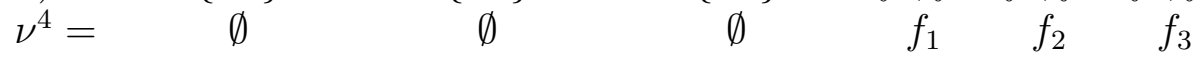

Note that $\nu^{i}, i=0, \ldots 4$, in $\mathcal{V}$ are not matchings. The algorithm cycles because $\nu^{0}=\nu^{4}$. It is easy to verify that $S^{*}(P)=\emptyset$, so the T-algorithm must cycle.

\section{The lattice structure of the core}

We shall introduce a partial order on $\mathcal{V}$ such that, if preferences are substitutable, $T$ is a monotone increasing map. Tarski's fixed point theorem then delivers a lattice structure on $\mathcal{E}$, and thus on $C_{w}(P)$.

Definition 4 Define the following partial orders on $\mathcal{V}_{F}, \mathcal{V}_{W}$ and $\mathcal{V}$ :

1. $P(F)$ on $\mathcal{V}_{F}$ by $\nu_{F} P(F) \nu_{F}^{\prime}$ if and only if $\nu_{F}^{\prime} \neq \nu_{F}$ and, for all $f$ in $F, \nu_{F}(f)=$ $\nu_{F}^{\prime}(f)$ or

$$
\nu_{F}(f)=C h\left(\nu_{F}(f) \cup \nu_{F}^{\prime}(f), P(f)\right) .
$$

2. $P(W)$ on $\mathcal{V}_{W}$ by $\nu_{W} P(W) \nu_{W}^{\prime}$ if and only if $\nu_{W}^{\prime} \neq \nu_{W}$ and

$$
\nu_{W}(w) R(w) \nu_{W}^{\prime}(w) \text { for all } w \in W .
$$


3. The weak partial orders associated to $P(F)$ and $P(W)$ are denoted $R(F)$ and $R(W)$, defined as: $\nu_{F} R(F) \nu_{F}^{\prime}$ if $\nu_{F}=\nu_{F}^{\prime}$ or $\nu_{F} P(F) \nu_{F}^{\prime}$, and $\nu_{W} R(W) \nu_{W}^{\prime}$ if $\nu_{W}=\nu_{W}^{\prime}$ or $\nu_{W} P(W) \nu_{W}^{\prime}$.

4. $P(F)$ on $\mathcal{V}$ by $\nu P(F) \nu^{\prime}$ iff $\nu_{F} P(F) \nu_{F}^{\prime}$ and $\nu_{W}^{\prime} R(W) \nu_{W}$, or $\nu_{F} R(F) \nu_{F}^{\prime}$ and $\nu_{W}^{\prime} P(W) \nu_{W}$.

5. $P(W)$ on $\mathcal{V}$ by $\nu P(W) \nu^{\prime}$ iff $\nu_{W} P(W) \nu_{W}^{\prime}$ and $\nu_{F}^{\prime} R(F) \nu_{F}$, or $\nu_{W} R(W) \nu_{W}^{\prime}$ and $\nu_{F}^{\prime} P(F) \nu_{F}$.

Definition 5 A firm $f$ 's preference ordering $P(f)$ satisfies substitutability if for any set $S \subseteq W$ containing workers $w$ and $\bar{w}(w \neq \bar{w})$, if $w \in C h(S, P(f))$ then $w \in$ $C h(S \backslash\{\bar{w}\}, P(f))$. A preference profile $P$ is substitutable if, for each firm $f$, the preference ordering $P(f)$ satisfies substitutability.

Theorem 13 Let $P$ be substitutable. Then $\left(C_{w}(P), P(F)\right)$ and $\left(C_{w}(P), P(W)\right)$ are nonempty complete lattices, and

$$
\begin{aligned}
& \text { 1. } \inf _{P(F)} C_{w}(P)=\sup _{P(W)} C_{w}(P) \text {, } \\
& \text { 2. } \sup _{P(F)} C_{w}(P)=\inf _{P(W)} C_{w}(P) \text {. }
\end{aligned}
$$

Theorem 13 says that there are two distinguished core matchings, $\mu_{W}$ and $\mu_{F}$ such that: For firms, $\mu_{W}=\inf _{P(F)} C_{w}(P)$ is worse, and $\mu_{F}=\sup _{P(F)} C_{w}(P)$ is better, than any other core matchings. For workers $\mu_{W}=\sup _{P(W)} C_{w}(P)$ is better, and $\mu_{F}=$ $\inf _{P(W)} C_{w}(P)$ is worse, than any other core matching.

Proof of Theorem 13. We shall present the proof as 4 simple lemmata. We assume that $P$ is substitutable in the rest of the section.

Notation. Let $\mathcal{V}^{\prime}=\{\nu \in \mathcal{V}: \nu(s) R(s) \emptyset$, for all $s \in F \cup W\}$.

Remark. For all $\nu \in \mathcal{V}, T \nu \in \mathcal{V}^{\prime}$

Lemma $14\left(\mathcal{V}^{\prime}, P(F)\right)$ is a complete lattice.

Proof. For each $f \in F$, let $X_{f}=\{A \subseteq W: A R(f) \emptyset\}$, and let $\geq_{f}$ be the partial order on $X_{f}$ defined by $A \geq B$ iff $A=B$ or $A=C h(A \cup B, P(f)$ ). By Blair (1988) (see also Alkan $(2002)),\left(X_{f}, \geq_{f}\right)$ is a complete lattice.

For each $w$, let $X_{w}=\{f \in F: f R(w) \emptyset\} ;\left(X_{w}, R(w)\right)$ is a totally ordered finite set, so it is a complete lattice.

Now, $\mathcal{V}^{\prime}=\left(\times_{f \in F} X_{f}\right) \times\left(\times_{w \in W} X_{w}\right)$, and $P(F)$ is the product order of the partial orders introduced above. Hence $\left(\mathcal{V}^{\prime}, P(F)\right)$ is a complete lattice. 
Lemma 15 Let $\mu$ and $\mu^{\prime}$ be pre-matchings. If $\mu^{\prime} R(F) \mu$ then, for all $w \in W$ and $f \in F$,

$$
\begin{aligned}
& U(f, \mu) \subseteq U\left(f, \mu^{\prime}\right) \\
& V(w, \mu) \supseteq V\left(w, \mu^{\prime}\right)
\end{aligned}
$$

Proof. Let $w \in U(f, \mu)$. We have that $f R(w) \mu(w)$, but $\mu(w) R(w) \mu^{\prime}(w)$ so $w \in$ $U\left(f, \mu^{\prime}\right)$. This proves $U(f, \mu) \subseteq U\left(f, \mu^{\prime}\right)$.

Now we shall prove that $V(w, \mu) \supseteq V\left(w, \mu^{\prime}\right)$. First, if $V\left(w, \mu^{\prime}\right)=\{\emptyset\}$, then there is nothing to prove, as $\{\emptyset\}=V\left(w, \mu^{\prime}\right) \subseteq V(w, \mu)$. Suppose that $V\left(w, \mu^{\prime}\right) \neq\{\emptyset\}$, and let $f \in V\left(w, \mu^{\prime}\right)$. Then,

$$
w \in C h\left(\mu^{\prime}(f) \cup\{w\}, P(f)\right) .
$$

But $\mu^{\prime} R(F) \mu$, so the definition of $R(F)$ implies that, for all $f \in F$, either $\mu^{\prime}(f)=\mu(f)$ so $w \in C h(\mu(f) \cup\{w\}, P(f))$, or

$$
\mu^{\prime}(f)=C h\left(\mu^{\prime}(f) \cup \mu(f), P(f)\right) .
$$

Then statement (37) implies that

$$
\begin{aligned}
w & \in C h\left(\mu^{\prime}(f) \cup\{w\}, P(f)\right) \\
& =C h\left(C h\left(\mu^{\prime}(f) \cup \mu(f), P(f)\right) \cup\{w\}, P(f)\right) \\
& \stackrel{(1)}{=}
\end{aligned}
$$

Equality (1) is from Proposition 2.3 of Blair (1988) (He showed that, if $P$ is substitutable, then $C h(A \cup B, P(f))=C h(C h(A, P(f)) \cup B, P(f))$ for all $A$ and $B)$. Now, substitutability of $P$ implies that

$$
w \in C h(\mu(f) \cup\{w\}, P(f)) .
$$

This means that $f \in V(w, \mu)$, thus completing the proof that $V(w, \mu) \supseteq V\left(w, \mu^{\prime}\right)$.

Lemma $16 \mathcal{E}$ is a nonempty and complete lattice.

Proof. First we show that $\left.T\right|_{\mathcal{V}^{\prime}}$ is monotone increasing. That is, whenever $\mu^{\prime} R(F) \mu$, we have $\left(T \mu^{\prime}\right) R(F)(T \mu)$.

Let $\mu^{\prime} R(F) \mu$, and fix $f \in F$ and $w \in W$. Lemma 15 says that $U(f, \mu) \subseteq U\left(f, \mu^{\prime}\right)$. We first show that

$$
C h\left(U\left(f, \mu^{\prime}\right), P(f)\right)=C h\left(\left[C h\left(U\left(f, \mu^{\prime}\right), P(f)\right) \cup C h(U(f, \mu), P(f))\right], P(f)\right) .
$$

To see this, let $S \subseteq C h\left(U\left(f, \mu^{\prime}\right), P(f)\right) \cup C h(U(f, \mu), P(f))$. Then $S \subseteq U(f, \mu) \cup$ $U\left(f, \mu^{\prime}\right)=U\left(f, \mu^{\prime}\right)$, so $C h\left(U\left(f, \mu^{\prime}\right), P(f)\right) R(f) S$. But, $C h\left(U\left(f, \mu^{\prime}\right), P(f)\right) \subseteq C h\left(U\left(f, \mu^{\prime}\right), P(f)\right) \cup$ $C h(U(f, \mu), P(f))$, so we have established statement 38 . 
Now, $\left(T \mu^{\prime}\right)(f)=C h\left(U\left(f, \mu^{\prime}\right), P(f)\right)$ and $(T \mu)(f)=C h(U(f, \mu), P(f))$, so statement 38 implies that

$$
\left.\left(T \mu^{\prime}\right)(f)=C h\left(\left[\left(T \mu^{\prime}\right)(f) \cup(T \mu)(f)\right], P(f)\right)\right) .
$$

We now show that $(T \mu)(w) R(w)\left(T \mu^{\prime}\right)(w)$. Lemma 15 says that $V\left(w, \mu^{\prime}\right) \subseteq V(w, \mu)$. So,

$$
(T \mu)(w)=\max _{P(f)}\{V(w, \mu)\} R(w) \max _{P(f)}\left\{V\left(w, \mu^{\prime}\right)\right\}=\left(T \mu^{\prime}\right)(w) .
$$

Statements 39 and 40 imply that $\left(T \mu^{\prime}\right) R(F)(T \mu)$, as $f \in F$ and $w \in W$ were arbitrary.

Finally, $T(\mathcal{V}) \subseteq \mathcal{V}^{\prime}$ so $\mathcal{E} \subseteq \mathcal{V}^{\prime}$, and $\mathcal{E}$ equals the set of fixed points of $\left.T\right|_{\mathcal{V}^{\prime}} . T(\mathcal{V}) \subseteq \mathcal{V}^{\prime}$ also implies that the restricted map $\left.T\right|_{\mathcal{V}^{\prime}}$ has range in $\mathcal{V}^{\prime}$.

Now we have that $\left.T\right|_{\mathcal{V}^{\prime}}: \mathcal{V}^{\prime} \rightarrow \mathcal{V}^{\prime}$ is monotone increasing, and $\mathcal{V}^{\prime}$ is a complete lattice by Lemma 14. So Tarski's fixed point theorem implies that $(\mathcal{E}, P(F))$ is a non-empty complete lattice.

Lemma $17\left(C_{w}(P), P(F)\right)$ and $\left(C_{w}(P), P(W)\right)$ are non-empty complete lattices, and

$$
\begin{aligned}
& \text { 1. } \inf _{P(F)} C_{w}(P)=\sup _{P(W)} C_{w}(P) \text {, } \\
& \text { 2. } \sup _{P(F)} C_{w}(P)=\inf _{P(W)} C_{w}(P) \text {. }
\end{aligned}
$$

Proof. By Corollary 5 and Lemma 16 we are done with $\left(C_{w}(P), P(F)\right)$. The orders $P(F)$ and $P(W)$ are order-duals, (Topkis (1998)) hence $\left(C_{w}(P), P(W)\right)$ is a non-empty complete lattice, and statements 1 and 2 in the lemma are immediate.

\section{The $T$-algorithm when preferences are substitutable.}

When preferences are substitutable, the $T$-algorithm

1. finds the worker-optimal core matching $\left(\inf _{P(F)} C_{w}(P)\right)$ and the firm-optimal core matching $\left(\sup _{P(F)} C_{w}(P)\right)$,

2. is computationally efficient - we can give a bound on its computational complexity and show that the bound is likely to be quite small,

3. calculates the lattice operations "join" and "meet," on the $C_{w}(P)$ lattice.

Result 1 is important because it shows that the $T$-algorithm does the job of the DAA. Further, by result 2 we have an idea of the $T$-algorithm's computational complexity, which is an open problem for the DAA in the many-to-one case. In a very simple example, we 
show that, while the "size" of the problem is 30,000 iterations, the $T$-algorithm needs at most 9 iterations to find a core matching. Our result on the complexity of the $T$ algorithm is similar to Topkis's (1979, see also Topkis (1998)) results on algorithms for finding equilibria in supermodular games.

Result 3: A number of papers have sought to improve on Blair's lattice operations (Martínez, Massó Neme, and Oviedo (2000) and Alkan (2002)) by restricting firms' preferences. We show that, with only substitutability, the $T$-algorithm can compute the lattice operations - thus allowing for a practical solution to the problem of Blair's lattice operations.

\subsection{The firm- and worker-optimal core matchings}

Proposition 18 If $P$ is substitutable, the $T$-algorithm starting at $\inf _{P(F)} \mathcal{V}^{\prime}$ stops at the worker-optimal core matching $\inf _{P(F)} C_{w}(P)$; the T-algorithm starting at $\sup _{P(F)} \mathcal{V}^{\prime}$ stops at the firm-optimal core matching $\sup _{P(F)} C_{w}(P)$.

Proof. When preferences are substitutable, $T$ is a monotone map on the complete lattice $\mathcal{V}^{\prime}$. Proposition 18 follows then from standard results, see Topkis (1979) or Topkis (1998).

\section{Remarks.}

1. The pre-matching $\inf _{P(F)} \mathcal{V}^{\prime}$ is $\nu \in \mathcal{V}$ with $\nu(f)=\{\emptyset\}$ and

$$
\nu(w)=\max _{P(w)} F
$$

The pre-matching $\sup _{P(F)} \mathcal{V}^{\prime}$ is $\nu \in \mathcal{V}$ with $\nu(f)=C h(W, P(f))$ and $\nu(w)=\{\emptyset\}$.

2. The $T$-algorithm starting at points that are not $\inf _{P(F)} \mathcal{V}^{\prime}$ or $\sup _{P(F)} \mathcal{V}^{\prime}$ may not converge. For example, one can show that the $T$-algorithm starting at the prematching

$$
\nu=\left(\left(\sup _{P(F)} \mathcal{V}^{\prime}\right)_{F},\left(\inf _{P(F)} \mathcal{V}^{\prime}\right)_{W}\right)
$$

cycles.

\subsection{Complexity of the $T$-algorithm}

We shall now give a bound on the computational complexity of the $T$-algorithm, but we need some auxiliary definitions first. 
For each $f$, let $X_{f}=\{A \subseteq W: A R(f) \emptyset\}$, and let $\geq_{f}$ be the partial order on $X_{f}$ defined by $A \geq B$ iff $A=C h(A \cup B, P(f))$ or $A=B$. Let $q_{f}$ be the size of the longest $\geq_{f^{-}}$chain in $X_{f}$. For each $w$, let $X_{w}=\{f \in F: f R(w) \emptyset\}$, and let $q_{w}$ be the number of elements in $X_{w}$.

To see what the definitions mean, consider the following example.

Example $19 F=\left\{f_{1}, f_{2}, f_{3}\right\}$, and $W=\left\{w_{1}, w_{2}, w_{3}\right\}$.

$$
\begin{aligned}
& P\left(f_{1}\right)=\left\{w_{1}, w_{3}\right\},\left\{w_{1}, w_{2}\right\},\left\{w_{2}, w_{3}\right\},\left\{w_{1}\right\},\left\{w_{2}\right\},\left\{w_{3}\right\} \\
& P\left(f_{2}\right)=\left\{w_{1}, w_{3}\right\},\left\{w_{2}, w_{3}\right\},\left\{w_{1}, w_{2}\right\},\left\{w_{3}\right\},\left\{w_{2}\right\},\left\{w_{1}\right\} \\
& P\left(f_{3}\right)=\left\{w_{1}, w_{3}\right\},\left\{w_{1}, w_{2}\right\},\left\{w_{2}, w_{3}\right\},\left\{w_{1}\right\},\left\{w_{2}\right\},\left\{w_{3}\right\} \\
& P\left(w_{1}\right)=f_{1}, f_{2}, \\
& P\left(w_{2}\right)=f_{2}, f_{1}, \\
& P\left(w_{3}\right)=f_{1}, f_{2} .
\end{aligned}
$$

Consider firm $f_{1}: X_{f_{1}}=\left\{\left\{w_{1}, w_{3}\right\},\left\{w_{1}, w_{2}\right\},\left\{w_{2}, w_{3}\right\},\left\{w_{1}\right\},\left\{w_{2}\right\},\left\{w_{3}\right\}\right\}$. The problem is simple enough that we can enumerate all chains in $X_{f_{1}}$ :

$$
\begin{aligned}
& \left\{w_{1}, w_{3}\right\}>_{f_{1}}\left\{w_{1}, w_{2}\right\}>_{f_{1}}\left\{w_{1}\right\} \\
& \left\{w_{1}, w_{3}\right\}>_{f_{1}}\left\{w_{1}, w_{2}\right\}>_{f_{1}}\left\{w_{2}\right\} \\
& \left\{w_{1}, w_{3}\right\}>_{f_{1}}\left\{w_{2}, w_{3}\right\}>_{f_{1}}\left\{w_{2}\right\} \\
& \left\{w_{1}, w_{3}\right\}>_{f_{1}}\left\{w_{2}, w_{3}\right\}>_{f_{1}}\left\{w_{3}\right\} \\
& \left\{w_{1}, w_{3}\right\}>_{f_{1}}\left\{w_{1}\right\} \\
& \left\{w_{1}, w_{3}\right\}>_{f_{1}}\left\{w_{2}\right\} \\
& \left\{w_{1}, w_{3}\right\}>_{f_{1}}\left\{w_{3}\right\} \\
& \left\{w_{1}, w_{2}\right\}>_{f_{1}}\left\{w_{1}\right\} \\
& \left\{w_{1}, w_{2}\right\}>_{f_{1}}\left\{w_{2}\right\} \\
& \left\{w_{2}, w_{3}\right\}>_{f_{1}}\left\{w_{2}\right\} \\
& \left\{w_{2}, w_{3}\right\}>_{f_{1}}\left\{w_{3}\right\}
\end{aligned}
$$

To see why these are all chains, note that $\left\{w_{1}, w_{3}\right\}>_{f_{1}} B$ for all $B \in X_{f_{1}} \backslash\left\{\left\{w_{1}, w_{3}\right\}\right\}$; that $\left\{w_{1}, w_{2}\right\} \ngtr_{f_{1}}\left\{w_{2}, w_{3}\right\}$ and $\left\{w_{1}, w_{2}\right\} \ngtr_{f_{1}}\left\{w_{3}\right\}$, but that $\left\{w_{1}, w_{2}\right\}>_{f_{1}}\left\{w_{1}\right\}$ and $\left\{w_{1}, w_{2}\right\}>_{f_{1}}\left\{w_{2}\right\}$. Similarly with $\left\{w_{2}, w_{3}\right\}$ in place of $\left\{w_{1}, w_{2}\right\}$. Finally, there are no $A, B$ in $\left\{\left\{w_{1}\right\},\left\{w_{2}\right\},\left\{w_{3}\right\}\right\}$ with $A>_{f_{1}} B$.

Hence, $q_{f_{1}} \leq 3$. By similar calculations for firms $f_{2}$ and $f_{3}, q_{f} \leq 3$ for all $f \in F$. For workers, the size of the longest chain in $\left\{f_{1}, f_{2}, f_{3}\right\}$ is $q_{w}=2$, as $X_{w}$ has only got 2 elements.

Proposition 20 If $P$ is substitutable, the T-algorithm starting at $\inf _{P(F)} \mathcal{V}^{\prime}\left(\sup _{P(F)} \mathcal{V}^{\prime}\right)$ stops at the worker-optimal (firm-optimal) core matching in less than

$$
\sum_{s \in F \cup W}\left(q_{s}-1\right)
$$

iterations. 
Proof. Let $\nu^{0}, \nu^{1}, \ldots \nu^{k}$ be the sequence generated by the $T$-algorithm before it stops at a core matching $\nu^{k}$. The number of iterations before the algorithm stops at $\nu^{k}$ is $k$, we shall prove that $k \leq \sum_{s \in F \cup W}\left(q_{s}-1\right)$.

Assume - without loss of generality - that we started the $T$-algorithm at $\nu^{0}=\inf _{P(F)} \mathcal{V}^{\prime}$. Since $\nu^{1}=\left(T \nu^{0}\right) P(F) \nu^{0}$ and $T$ is monotone increasing (see the proof of Theorem 13),

$$
\nu^{k} P(F) \nu^{k-1} P(F) \ldots \nu^{1} P(F) \nu^{0} .
$$

For each $l, 1 \leq l \leq k$, it must be that $\nu^{l}(f)=\nu^{l-1}(f)$ or that $\nu^{l}(f)=C h\left(\nu^{l}(f) \cup\right.$ $\left.\nu^{l-1}(f), P(f)\right)$ for all $f$, and $\nu^{l-1}(w) R(w) \nu^{l}(w)$ for all $w$. Also, for each $l, 1 \leq l \leq k$, either $\nu^{l}(f) \neq \nu^{l-1}(f)$ and $\nu^{l}(f)=C h\left(\nu^{l}(f) \cup \nu^{l-1}(f), P(f)\right)$ for some for $f$ or $\nu^{l-1}(w) P(w) \nu^{l}(w)$ for some $w$-recall that $P(F)$ is a strict order, see Definition 4. Say that $l$ is an $f$-step if $\nu^{l}(f) \neq \nu^{l-1}(f)$ and $\nu^{l}(f)=C h\left(\nu^{l}(f) \cup \nu^{l-1}(f), P(f)\right)$ for firm $f$. Say that $l$ is a $w$-step if $\nu^{l-1}(w) P(w) \nu^{l}(w)$ for worker $w$. There cannot be more than $q_{s}-1 s$-steps, as a number of, say $r$ steps involves a chain of $r+1$ elements in $X_{s}$. Now $k$ is smaller than the sum over all $s \in W \cup F$ of all $s$-steps, so

$$
k \leq \sum_{s \in F \cup W}\left(q_{s}-1\right) .
$$

But $k$ is the number of iterations of the $T$-algorithm before it finds $\nu^{k}$.

Remark. Recall that $|W|=m$ and $|F|=n$, so the number of pre-matchings is

$$
2^{n m}(n+1)^{m},
$$

a number which is normally orders of magnitude larger than the bound in Proposition 20: In Example 19, Proposition 20 implies that the $T$-algorithm converges before

$$
\sum_{s \in F \cup W}\left(q_{s}-1\right)=9
$$

iterations. Compare with the number of pre-matchings: 32768. The number of matchings is smaller than the number of pre-matchings, but still substantially larger than 9.It may be unfair to compare the complexity of the $T$-algorithm to the number of prematchings, after all the $T$-algorithm will not involve pre-matchings where agents are matched to unacceptable partners - but still, the number of pre-matchings that only involve acceptable partners is 9261 .

\subsection{Computing the lattice operations}

Let $\mu^{1}, \mu^{2} \in C_{w}(P)$. We shall look at the problem of finding $\mu^{1} \vee_{P(F)} \mu^{2}$, the join (least upper bound) of $\mu^{1}$ and $\mu^{2}$ in the $\left(C_{w}(P), P(F)\right)$-lattice. One can solve the problem of finding $\mu^{1} \wedge_{P(F)} \mu^{2}$ in the $\left(C_{w}(P), P(F)\right)$-lattice in a similar way. 
Martínez, Massó, Neme and Oviedo (2000 and 2001) prove that, when preferences satisfy substitutability and an additional assumption (which they call $q$-separability) there is a simple formula for $\mu^{1} \vee_{P(F)} \mu^{2}$ (see Alkan (2002) for a similar result). Martínez, Massó, Neme and Oviedo prove that $\mu^{1} \vee_{P(F)} \mu^{2}$ is

$$
\bar{\nu}(s)= \begin{cases}C h\left(\mu^{1}(s) \cup \mu^{2}(s), P(s)\right) & \text { if } s \in F \\ \min _{P(s)}\left\{\mu^{1}(s), \mu^{2}(s)\right\} & \text { if } s \in W .\end{cases}
$$

In general, though, when preferences are only substitutable, there is no formula for $\mu^{1} \vee_{P(F)} \mu^{2}$. In fact, $\bar{\nu}$ defined in (41) is a pre-matching but not necessarily a matching. We prove that, if one starts the $T$-algorithm at $\bar{\nu}$, it will stop at $\mu^{1} \vee_{P(F)} \mu^{2}$. Hence we give a practical solution to Blair's problem of calculating $\mu^{1} \vee_{P(F)} \mu^{2} .^{3}$

Theorem 21 Let $P$ be substitutable, let $\mu^{1}$ and $\mu^{2}$ be core matchings, and $\bar{v}$ be defined as in (41). The T-algorithm started at $\bar{v}$ stops at $\mu^{1} \vee_{P(F)} \mu^{2}$.

Proof. We shall present the proof as two lemmata.

Lemma 22 Let $P$ be substitutable, and let

$$
\hat{V}=\{\nu \in V: \nu R(F) \bar{\nu}\}
$$

Then $T \hat{V} \subseteq \hat{V}$

Proof. First we shall prove that $\bar{\nu}$ is the least upper bound on $\left\{\mu^{1}, \mu^{2}\right\}$ in $\mathcal{V}$. By the definition of $\bar{\nu}, \bar{\nu} R(F) \mu^{1}$ and $\bar{\nu} R(F) \mu^{2}$.

Let $\nu$ be an upper bound on $\left\{\mu^{1}, \mu^{2}\right\}$ in $\mathcal{V}$, we shall prove that $\nu R(F) \bar{\nu}$. For any $f \in F:$

$$
\begin{aligned}
& \nu(f) R(f) \mu^{1}(f) \Longleftrightarrow \nu(f) \stackrel{(1)}{=} C h\left(\nu(f) \cup \mu^{1}(f), P(f)\right) \\
& \nu(f) R(f) \mu^{2}(f) \Longleftrightarrow \nu(f) \stackrel{(2)}{=} C h\left(\nu(f) \cup \mu^{2}(f), P(f)\right) .
\end{aligned}
$$

We have that

$$
\begin{aligned}
C h(\nu(f) \cup \bar{\nu}(f), P(f)) & \stackrel{(3)}{=} C h\left(\nu(f) \cup C h\left(\mu^{1}(f) \cup \mu^{2}(f), P(f)\right), P(f)\right) \\
& \stackrel{(4)}{=} C h\left(\nu(f) \cup \mu^{1}(f) \cup \mu^{2}(f), P(f)\right) \\
& \stackrel{(5)}{=} C h\left(C h\left(\nu(f) \cup \mu^{1}(f), P(f)\right) \cup \mu^{2}(f), P(f)\right) \\
& \stackrel{(6)}{=} C h\left(\nu(f) \cup \mu^{2}(f), P(f)\right) \\
& \stackrel{(7)}{=} \nu(f) .
\end{aligned}
$$

\footnotetext{
${ }^{3}$ Blair's proof of the existence of $\mu^{1} \vee_{P(F)} \mu^{2}$ can also be expressed as an algorithm for calculating $\mu^{1} \vee_{P(F)} \mu^{2}$.
} 
Where equality (3) follows from the definition of $\bar{\nu}$. Equalities (4) and (5) follow from substitutability and Proposition 2.3 of Blair (1988). Equality (6) follows from equality (1). Equality (7) follows from equality (2). Thus,

$$
\nu_{F} R(F) \bar{\nu}_{F} .
$$

Now, $\nu R(F) \mu_{1}$ and $\nu R(F) \mu_{2}$ imply that, for any $w \in W$,

$$
\begin{aligned}
& \mu_{1}(w) R(w) \nu(w), \text { and } \\
& \mu_{2}(w) R(w) \nu(w) .
\end{aligned}
$$

But then,

$$
\bar{\nu}(w)=\min _{P(w)}\left\{\mu^{1}(w), \mu^{2}(w)\right\} R(w) \nu(w) .
$$

This and condition (42) implies that $\nu R(F) \bar{\nu}$. Hence $\bar{\nu}$ is the least upper bound on $\left\{\mu^{1}, \mu^{2}\right\}$ in $\mathcal{V}$.

Now we shall prove that $T \hat{V} \subseteq \hat{V}$. Let $\nu \in \hat{V}$, then $\nu R(F) \bar{\nu} R(F) \mu^{1}$ and $\nu R(F) \bar{\nu} R(F) \mu^{2}$. But then $T \nu R(F) T \mu^{1}$ and $T \nu R(F) T \mu^{2}$, as $T$ is monotone. Now, $\mu^{1}$ and $\mu^{2}$ are core matchings, so $\mu^{1}=T \mu^{1}$ and $\mu^{2}=T \mu^{2}$; then $T \nu$ is an upper bound on $\left\{\mu^{1}, \mu^{2}\right\}$ in $\mathcal{V}$. But $\bar{\nu}$ is the least upper bound, so $T \nu R(F) \bar{\nu}$ and thus $T \nu \in \hat{V}$.

Lemma 23 The T-algorithm starting at $\bar{\nu}$ stops at $\mu^{1} \vee_{P(F)} \mu^{2}$.

Proof. By Lemma 22, T| $\left.\right|_{\hat{V}}: \hat{V} \rightarrow \hat{V}$. T is monotone and $\bar{\nu}$ is the smallest pre-matching in $\hat{V}$. By Topkis's (1979) results, the $T$-algorithm stops at the $R(F)$-smallest fixed point of $\left.T\right|_{\hat{V}}$; but this is the $R(F)$-smallest fixed point of $T$, of those fixed points that are $R(F)$-larger than both $\mu^{1}$ and $\mu^{2}$. Then the $T$-algorithm stops at $\mu^{1} \vee_{P(F)} \mu^{2}$.

\section{Remarks.}

1. A key part of the proof of Theorem 21 is that $\bar{\nu}$ is the join of $\mu^{1}$ and $\mu^{2}$ in $\mathcal{V}$. By a similar argument, the $T$-algorithm calculates the meet, $\mu^{1} \wedge_{P(F)} \mu^{2}$, of any two core matchings $\mu^{1}$ and $\mu^{2}$. One only needs to start the algorithm at the meet of $\mu^{1}$ and $\mu^{2}$ in $\mathcal{V}$ instead of the join. ${ }^{4}$

2. The bound presented in Proposition 20 also bounds the number of $T$-iterations necessary to calculate $\mu^{1} \vee_{P(F)} \mu^{2}$.

We illustrate how the algorithm finds the join of two core matchings using an example from Roth (1985).

\footnotetext{
${ }^{4}$ See Alkan (2002) on how to calculate the meet of $\mu^{1}$ and $\mu^{2}$ in $\mathcal{V}$.
} 
Example 24 (Roth 1985). Let $F=\left\{f_{1}, f_{2}, f_{3}, f_{4}, f_{5}\right\}$ be the set of firms and $W=$ $\left\{w_{1}, w_{2}, w_{3}, w_{4}, w_{5}, w_{6}\right\}$ be the set of workers. As in Roth (1985), it will not be necessary to specify the full preference ordering of each agent, since they may be extended in several ways and still preserve the substitutability of the firms' preferences. The preference profile is as follows:

$$
\begin{aligned}
& P\left(f_{1}\right)=\left\{w_{4}\right\},\left\{w_{1}\right\},\left\{w_{2}, w_{3}, w_{5}, w_{6}\right\}, \ldots,\left\{w_{5}\right\}, \ldots \\
& P\left(f_{2}\right)=\left\{w_{2}\right\},\left\{w_{1}, w_{3}\right\}, \ldots \\
& P\left(f_{3}\right)=\left\{w_{3}\right\},\left\{w_{2}\right\}, \ldots \\
& P\left(f_{4}\right)=\left\{w_{5}\right\},\left\{w_{4}, w_{6}\right\}, \ldots \\
& P\left(f_{5}\right)=\left\{w_{6}\right\},\left\{w_{5}\right\}, \ldots \\
& P\left(w_{1}\right)=f_{2}, f_{1}, \ldots \\
& P\left(w_{2}\right)=f_{1}, f_{3}, f_{2}, \ldots \\
& P\left(w_{3}\right)=f_{1}, f_{2}, f_{3}, \ldots \\
& P\left(w_{4}\right)=f_{4}, f_{1}, \ldots \\
& P\left(w_{5}\right)=f_{1}, f_{5}, f_{4}, \ldots \\
& P\left(w_{6}\right)=f_{1}, f_{4}, f_{5}, \ldots
\end{aligned}
$$

Consider the following two core matchings

$$
\begin{aligned}
& \mu_{1}=\left\{\begin{array}{cccccc}
f_{1} & f_{2} & f_{3} & f_{4} & f_{5} & \\
& \left\{w_{1}\right\} & \left\{w_{2}\right\} & \left\{w_{3}\right\} & \left\{w_{4}, w_{6}\right\} & \left\{w_{5}\right\}
\end{array}\right. \text { and } \\
& \mu_{2}=\left\{\begin{array}{ccccc}
f_{1} & f_{2} & f_{3} & f_{4} & f_{5} \\
\left\{w_{4}\right\} & \left\{w_{1}, w_{3}\right\} & \left\{w_{2}\right\} & \left\{w_{5}\right\} & \left\{w_{6}\right\}
\end{array} .\right. \\
& \begin{array}{lllllllllll}
f_{1} & f_{2} & f_{3} & f_{4} & f_{5} & w_{1} & w_{2} & w_{3} & w_{4} & w_{5} & w_{6}
\end{array}
\end{aligned}
$$

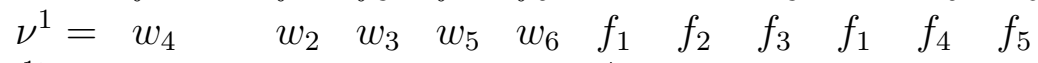

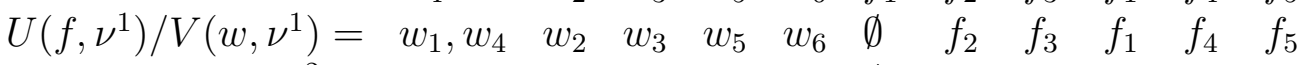

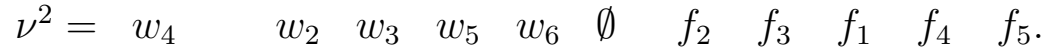

$\nu^{2}$ is a matching and $\nu^{2}=\mu_{1} \vee_{P(F)} \mu_{2}$.

\section{References}

H. Adachi (2000). "On a Characterization of Stable Matchings," Economic Letters. 68, 43-49.

A. Alkan (2002). "A Class of Multipartner Matching Markets with a Strong Lattice Structure", Economic Theory 19, 737-746.

C. Blair (1988). "The Lattice Structure of the Set of Stable Matchings with Multiple Partners", Mathematics of Operations Research 13, 619-628.

T. Fleiner (2001). "A Fixed-point Approach to Stable Matchings and Some Applications", Technical Report, Egervary Research Group on Combinatorial Optimization. 
D. Gusfield and R. Irving (1989). The Stable Marriage Problem: Structure and Algorithms. Cambridge: MIT Press.

R. Martínez, J. Massó, A. Neme, and J. Oviedo (2000). "Single Agents and the Set of Many-to-one Stable Matchings", Journal of Economic Theory 91, 91-105.

R. Martínez, J. Massó, A. Neme, and J. Oviedo (2001). "On the Lattice Structure of the Set of Stable Matchings for a Many-to-one Model", Optimization 50, 439-457.

A. Roth (1985). "Conflict and Coincidence of Interest in Job Matching: Some New Results and Open Questions", Mathematics of Operations Research 10, 379-389.

A. Roth and M. Sotomayor (1988). "Interior Points in the Core of Two-Sided Matching Markets," Journal of Economic Theory. 45, 85-101.

A. Roth and M. Sotomayor (1990). Two-sided Matching: A Study in Game-Theoretic Modelling and Analysis. Cambridge University Press, Cambridge, England. [Econometrica Society Monographs No. 18].

M. Sotomayor (1999). "Three Remarks on the Many-to-many Stable Matching Problem", Mathematical Social Sciences 38, 55-70.

D. M. Topkis (1979). "Equilibrium Points in Non-zero sum n-person submodular games," SIAM Journal on Control and Optimization 17, 773-787.

D. M. Topkis (1998). Supermodularity and Complementarity. Princeton University Press.. 